\title{
A CHARACTERIZATION OF REFLEXIVE BANACH SPACES
}

\author{
EVA MATOUŠKOVÁ AND CHARLES STEGALL
}

(Communicated by Dale Alspach)

\begin{abstract}
A Banach space $Z$ is not reflexive if and only if there exist a closed separable subspace $X$ of $Z$ and a convex closed subset $Q$ of $X$ with empty interior which contains translates of all compact sets in $X$. If, moreover, $Z$ is separable, then it is possible to put $X=Z$.
\end{abstract}

We consider the following problem: When does a Banach space contain a closed convex set $Q$ with empty interior which contains a translate of any compact set in $X$ ? The basic example of such a Banach space is the space $C(\mathcal{K})$ of continuous functions on a compact infinite space $\mathcal{K}$. Indeed, it is enough to choose a point $p \in \mathcal{K}$ which is not isolated and define $Q$ as the set of all functions in $C(\mathcal{K})$ which attain their minima at $p$. Since $p$ is not isolated, $Q$ has empty interior. If $K$ is a compact subset of $C(\mathcal{K})$, then by the Banach-Dieudonné theorem [3] there exists a sequence $\left\{f_{n}\right\}$ of functions in $C(\mathcal{K})$ converging to zero such that $K$ is contained in its closed convex hull. If we define the function $g$ by

$$
g(t):=\sup \left\{\left|f_{n}(t)-f_{n}(p)\right|: n \in N\right\} \text { for } t \in \mathcal{K},
$$

then it is easy to check that $g$ is continuous and each function $g+f_{n}$ is in $Q$. Consequently, since $Q$ is convex, the translate $g+K$ is contained in $Q$.

If a Banach space $Z$ can be mapped linearly onto a Banach space $X$ containing the required set $Q$, then $Z$ also contains such a set. Namely, by the open mapping theorem, it is enough to take the preimage of $Q$. Therefore, for example, $\ell_{1}$ contains the required set because it can be mapped onto any separable Banach space, in particular, $C[0,1]$.

In this note we show that, in fact, any separable nonreflexive Banach space $X$ contains a closed convex set with empty interior which contains a translate of any compact set in $X$.

Borwein and Noll observed in [1] that there exist a convex continuous function on the space $c_{0}$ of null sequences and a closed subset $Q$ of $c_{0}$ which is not a Haar null set so that $f$ fails to be Fréchet differentiable on $Q$. They define $f$ as the distance from the positive cone $Q:=\left\{\left\{x_{n}\right\} \in c_{0} ; x_{n} \geq 0, n=1,2, \ldots\right\}$. As $Q$ has no interior points, $f$ fails to be Fréchet differentiable at all points of $Q$. The set $Q$ contains a translate of any compact set in $c_{0}$, and, therefore, for any

Received by the editors May 24, 1994 and, in revised form, August 18, 1994.

1991 Mathematics Subject Classification. Primary 46B10; Secondary 46B20.

Key words and phrases. Banach spaces, reflexivity, convexity.

The first author was partially supported by a grant of the Österreichische Akademische Austauschdienst. 
probability Borel measure $\mu$ on $c_{0}$ there exists some $x \in c_{0}$ such that $\mu(Q+x)>0$. Consequently, $Q$ is not Haar null (for the definition see [2]). They conjecture in [1] that also in $\ell_{2}$ there exists a closed convex set $C$ with empty interior which contains a translate of any compact set. We show that this is not the case in any reflexive Banach space, but on the other hand every nonreflexive Banach space has a closed subspace containing such a set.

By $B_{X}$ we denote the open unit ball of a Banach space $X$, and $B_{X}(x, r)$ is the usual notation for the open ball with center $x$ and diameter $r$; the subscript will be often omitted. We denote the closure of a set $A$ by $\bar{A}$ or $\operatorname{cl} A$.

We will make use of the following variation of the Banach-Dieudonné theorem: Let $X$ be a Banach space, $K$ a compact subset of $B_{X}(0, c)$ and $E$ a dense subset of $B_{X}(0,2 c)$. Then there exists a sequence $\left\{F_{n}\right\}$ of finite sets in $E$ so that

$$
K \subset \mathrm{cl}\left(\sum_{n=1}^{\infty} 2^{-n} F_{n}\right) .
$$

This follows from the fact that there exist a sequence $\left\{F_{n}\right\}$ of finite sets in $E$ and a sequence of compact sets $\left\{K_{n}\right\}$ in $\bar{B}_{X}(0, c)$ so that

$$
K \subset \sum_{i=1}^{n} 2^{-i} F_{i}+2^{-n} K_{n} \quad \text { for } n \in N .
$$

Indeed, if $n=1$, choose $F_{1} \subset E$ so that $2^{-1} F_{1}$ is a $\frac{c}{2}$-net for $K$. Then the set

$$
K_{1}:=2\left(\left(K-2^{-1} F_{1}\right) \cap \bar{B}\left(0, \frac{c}{2}\right)\right)
$$

is a compact subset of $\bar{B}(0, c)$ and $K \subset 2^{-1} F_{1}+2^{-1} K_{1}$. Now we can continue by induction. Suppose that $F_{i}$ and $K_{i}$ for $i=1, \ldots, n$ so that $(2)$ holds have been already constructed. Choosing $F_{n+1} \subset E$ so that $2^{-1} F_{n+1}$ is a $\frac{c}{2}$-net for the set $K_{n}$ and defining

$$
K_{n+1}:=2\left(\left(K_{n}-2^{-1} F_{n+1}\right) \cap \bar{B}\left(0, \frac{c}{2}\right)\right)
$$

completes the proof. The following lemmata are possibly not the most efficient way to our main result, but we think that they may be of independent interest.

Lemma 1. Let $Z$ be a Banach space, $U$ an open convex subset of $Z$ and $f$ a continuous real valued function defined on $U$. Then, either $f$ is affine or the convex hull $G$ of the graph of $f$ has nonempty interior.

Proof. Suppose that $f$ is not affine. Then there exist $x$ and $y$ in $U$ such that $1 / 2(f(x)+f(y)) \neq f((x+y) / 2)$. Define $z_{0}:=(x+y) / 2$ and $c:=(f(x)+f(y)) / 2$. We can suppose by replacing $f$ by $-f$ and adding a constant, if necessary, that

$$
(f(x)+f(y)) / 2-f\left(z_{0}\right)=\alpha>0 \text { and } f\left(z_{0}\right)>0 .
$$

Choose some $\varepsilon>0$ so that $0<f(v)<f\left(z_{0}\right)+\alpha / 2$ for every $v \in Z$ for which $\left\|v-z_{0}\right\|<\varepsilon$. Clearly the interior of the cone cap

$$
\begin{aligned}
M & :=\left\{x_{z, t}=t(z, 0)+(1-t)\left(z_{0}, c\right): z \in Z,\left\|z-z_{0}\right\|<\varepsilon, 0 \leq t \leq \alpha /(2 c)\right\} \\
& \subset Z \times R
\end{aligned}
$$

is nonempty. Let some $x_{z, t} \in M$ be given, we will show that $x_{z, t} \in G$. Consider the function

$$
g(s):=(1-s) c-f\left((1-s) z_{0}+s z\right), \quad t \leq s \leq 1
$$


The function $g$ is continuous, $g(t)>0$, and $g(1)<0$. Therefore, there exists some $r \in(t, 1)$ for which $g(r)=0$. Hence, $x_{z, r}$ is contained in the graph of $f$ and since

$$
x_{z, t}=\frac{t}{r} x_{z, r}+\left(1-\frac{t}{r}\right)\left(z_{0}, c\right)
$$

we have $x_{z, t} \in G$.

We say that a convex subset $Q$ of a Banach space $X$ is spanning if it contains a line segment in every direction, that is $X=\bigcup_{t>0} t(Q-Q)$. Observe that if a convex set $Q$ contains a translate of every finite subset of the unit ball, then $Q$ is spanning. If $Q$ contains translates of all compact sets in $X$ (or, for that matter, of all line segments), then $X=Q-Q$. Indeed, if $x \in X$ is given, then there exists $z \in X$ so that $[z, z+x] \subset Q$, and $x=z+x-z \in Q-Q$.

Lemma 2. Suppose that $X$ is a Banach space and $Q \subseteq X$ is a bounded, closed and convex set with empty interior that is also spanning. Then for any compact subset $K$ of $X$ it follows that $Q+K$ also has empty interior.

Proof. First, we show that $Q \cap H$ is nowhere dense in $H$ if $H$ is any closed hyperplane. Suppose that $x^{*} \neq 0, w \in H=\left\{x^{*}=a\right\}, \delta>0$ and

$$
B(w, \delta) \cap H \subseteq Q \cap H .
$$

Choose some $y \in X$ such that $\left\langle x^{*}, y\right\rangle>0$. Since $Q$ is spanning there exist $t>0, u$ and $v$, both in $Q$, so that $t(u-v)=y$. It follows that $\left\langle x^{*}, u-v\right\rangle>0$ and one of $u$ or $v$, say $u$, is not in $H$. It is routine to check that the convex hull of $\{u\} \cup(B(w, \delta) \cap H)$ has an interior point relative to $X\left(\operatorname{try} \frac{1}{2}(u+w)\right)$ which contradicts the fact that $Q$ has no interior. Suppose that $H \subseteq X$ is a closed hyperplane, $u \in X, x \notin H$ and suppose that $h^{*} \in H^{*}$. Then the set $\left\{y+\left\langle h^{*}, y\right\rangle x+u: y \in H\right\}$ is a hyperplane in $X$ and the transformation $y \mapsto y+\left\langle h^{*}, y\right\rangle x+u$ is an affine homeomorphism. If $x \in X$, then the set $Q^{\prime}=Q+[-x, x]$ is also bounded, closed, convex and spanning. We will show that it has empty interior. Suppose that the interior of $Q^{\prime}$ is nonempty. Then $x \neq 0$; choose $x^{*} \in X^{*}$ so that $\left\langle x^{*}, x\right\rangle>0$. Let $P$ be the projection on $X$ whose image is the kernel $H$ of $x^{*}$ and whose kernel is the span of $x$. The open mapping theorem says that $P(Q)=P\left(Q^{\prime}\right)$ has nonempty interior in $H$. Suppose that $w \in H, \delta>0$ and $B(w, \delta) \cap H \subseteq P(Q)$. For $z \in B(w, \delta) \cap H$ define

$$
f(z):=\inf \{t: z+t x \in Q\} .
$$

It is easy to see that $f$ is bounded and convex, hence continuous. The mapping $(z, t) \mapsto z+t x$ is an isomorphism from $H \times R$ onto $X$ which maps the graph of $f$ onto the set $\{z+f(t) x: z \in B(w, \delta) \cap H\} \subset Q$. Because $Q$ has empty interior, Lemma 1 implies that $f$ must be affine, and we shall show that this leads to a contradiction. Since it is defined on an open convex subset of $H$, there exists an $h^{*} \in H^{*}$ and a real number $b$ such that $f(z)=\left\langle h^{*}, z\right\rangle+b$. Finally,

$$
\left\{z+\left\langle h^{*}, z\right\rangle x+b x: z \in B(w, \delta) \cap H\right\} \subseteq Q
$$

and this means that $Q$ contains a relatively open subset of a hyperplane, which is a contradiction. By induction, given $x_{1}, x_{2}, \ldots, x_{n} \in X$ we have that

$$
Q+\left[-x_{1}, x_{1}\right]+\cdots+\left[-x_{n}, x_{n}\right]
$$

has no interior point. The case of an arbitrary compact set $K$ can be verified by an application of (1). We give a few details. Suppose the interior of $Q+K$ is nonempty. By translating $Q+K$ if necessary we can suppose that $B(0, r) \subset Q+K$ 
for some $r>0$. Choose a sequence $\left\{F_{n}\right\}$ of finite subsets of a ball in $X$ so that (1) holds. Choose $n_{0} \in N$ so that

$$
\sum_{i=n_{0}}^{\infty} 2^{-i} F_{i} \subset B(0, r / 4) .
$$

Because the interior of the closed and convex set $Q_{0}:=Q+\sum_{i=1}^{n_{0}} 2^{-i} \operatorname{co} F_{i}$ is empty, there exists $v \in B_{X}(0, r)$ so that

$$
\operatorname{dist}\left(v, Q_{0}\right)>r / 2 \text {. }
$$

To see this choose a point $y \in B(0, r / 4) \backslash Q_{0}$ and $x^{*}$ in the unit sphere of $X^{*}$ which separates $y$ from $Q_{0}$, namely $r / 4 \geq\left\langle x^{*}, y\right\rangle \geq\left\langle x^{*}, u\right\rangle$ for any $u \in Q_{0}$. Choose $x \in B(0, r)$ so that $\left\langle x^{*}, x\right\rangle>3 r / 4$. Then $v=x$ satisfies the required inequality. From (3) and (4) follows that

$$
\operatorname{dist}\left(v, Q+\sum_{i=1}^{\infty} 2^{-i} F_{i}\right) \geq r / 4,
$$

which is a contradiction.

With the hypothesis above, observe that if $T: X \rightarrow Y$ is a surjective linear operator with finite-dimensional kernel $F$, then $T(Q)$ is a bounded, closed and convex set with empty interior that is also spanning; this is because $T^{-1}(T(X))=Q+F$ is a first category set.

In connection with the next theorem observe that the positive cone of $\ell_{2}$ is a closed convex set with empty interior which contains a translate of any finite subset $F$ of $\ell_{2}$. (Indeed, if for $x=\left\{x_{n}\right\} \in \ell_{2}$ we define $x^{-}=\left\{x_{n}^{-}\right\}$so that $x_{n}^{-}=-x_{n}$ if $x_{n}<0$ and $x_{n}^{-}=0$ otherwise, then the set $F+\sum_{x \in F} x^{-}$is contained in the positive cone.) However, as we will see later, because $\ell_{2}$ is reflexive it does not contain a closed convex set with empty interior containing a translate of every compact set. Hence the boundedness hypothesis in (iv) of the next theorem is needed.

Theorem 3. Let $X$ be a Banach space. Then the following are equivalent:

(i) there exists a convex and closed subset $Q$ of $X$ with empty interior which contains translates of all compact sets in $X$; i.e. whenever $K$ is a compact subset of $X$ there exists $x_{K} \in X$ so that $K+x_{K} \subset Q$;

(ii) there exists a convex and closed subset $P$ of $X$ with empty interior such that if $K$ is a compact subset of the unit ball of $X$, then there exists $x_{K} \in X$ so that $K+x_{K} \subset P$

(iii) there exists a convex, closed and bounded subset $C$ of $X$ with empty interior such that if $K$ is a compact subset of the unit ball of $X$, then there exists $x_{K} \in X$ so that $K+x_{K} \subset C$; and

(iv) there exist a dense subset $E$ of the unit ball of $X$ and a convex, closed and bounded subset $D$ of $X$ with empty interior so that whenever $F$ is a finite set contained in $E$, there exists $x_{F} \in X$ so that $F+x_{F} \subset D$.

Proof. Clearly (i) implies (ii). To prove that (ii) implies (iii), it is enough to show that there exists $1 \geq r>0$ and $c>0$ so that for any compact set $K \subset \bar{B}(0, r)$ there exist $z_{K} \in B(\overline{0}, c)$ so that $K+z_{K} \subset P$, for then we may define

$$
C:=\frac{1}{r}(P \cap \bar{B}(0, r+c)) \text {. }
$$


For a contradiction, suppose that for every $n \in N$ there exists a compact set $K_{n} \subset \bar{B}(0,1 / n)$ so that

$$
\text { if } K_{n}+x \subset P \text {, then }\|x\| \geq n .
$$

Define

$$
K:=\bigcup_{n=1}^{\infty} K_{n} \cup\{0\}
$$

The set $K$ is a compact subset of the unit ball, hence there exists $z \in X$ such that $K+z \subset P$. Because $K_{n} \subset K$ for $n \in N$, we have $\|z\| \geq n$ for all $n$, which is nonsense.

Let us show now that (iii) implies (i). We can suppose that zero is not contained in $C$ and define

$$
Q:=\bigcup_{\lambda \geq 0} \lambda C
$$

The set $Q$ is convex and contains translates of all compact sets in $X$. To show that $Q$ is closed, let $z \in X, x_{n} \in C$ and $\lambda_{n} \geq 0$ such that $\lim _{n \rightarrow \infty} \lambda_{n} x_{n}=z$ be given. Because the sequence $\left\{x_{n}\right\}$ is bounded away from zero, the sequence $\left\{\lambda_{n}\right\}$ is bounded, and consequently it has a converging subsequence $\lambda_{n_{k}} \rightarrow \lambda \geq 0$. If $\lambda=0$, then from the boundedness of $C$ it follows that $z=0 \in Q$. Otherwise the sequence $\left\{x_{n_{k}}\right\}$ converges to $z / \lambda$. Because $C$ is closed we get that $z=\lim _{k \rightarrow \infty} \lambda_{n_{k}} x_{n_{k}}=z \in$ $\lambda C$. Finally, let us show that the set $Q$ has empty interior. Choose some $z \in C$. The set $\tilde{C}:=C+[-z, 0]$ is closed and convex, and because $C$ is spanning $\tilde{C}$ has empty interior by Lemma 2. Since

$$
Q=\bigcup_{\lambda \geq 0} \lambda C \subset \bigcup_{n \in N} n \tilde{C}
$$

it follows from the Baire theorem that the interior of $Q$ is empty.

Clearly (iii) implies (iv), so let us show that the opposite implication also holds. Let $K$ be a compact subset of $B_{X}\left(0,2^{-1}\right)$. We will show that $K$ can be translated into $D$. Then $C:=2 D$ will satisfy (iii). Let $\left\{F_{n}\right\}$ be a sequence of finite sets in $E$ so that (1) holds. Choose $z_{n} \in X$ so that $z_{n}+F_{n} \subset D$. Because $D$ is bounded, the sequence $\left\{z_{n}\right\}$ is bounded. If we define $z:=\sum_{n=1}^{\infty}\left(1 / 2^{n}\right) z_{n}$, we get

$$
z+K \subset z+\mathrm{cl} \sum_{n=1}^{\infty} 2^{-n} F_{n} \subset \mathrm{cl}\left(\sum_{n=1}^{\infty} 2^{-n} z_{n}+2^{-n} F_{n}\right) \subset D,
$$

where the last inclusion follows from the fact that $D$ is convex and closed.

It should be remarked here that from the proof of equivalence of (i) and (iii) of the previous theorem it follows that if a Banach space $X$ contains a closed and convex set with empty interior containing the translates of all compacts, then $X$ contains a closed and convex cone $Q$ with empty interior which contains the translates of all compacts.

Corollary 4. Let $Z$ be a Banach space and $Y$ be a separable subspace of $Z$. Let $Z$ contain a convex closed set $Q$ with empty interior which contains translates of all compact sets in $Z$. Then there exist a closed, separable and linear subspace $X$ of $Z$ containing $Y$ and a convex closed subset $P$ of $X$ with empty interior which contains translates of all compact sets in $X$. 
Proof. By Theorem 3 there exists a convex closed bounded subset $C$ of $Z$ with empty interior which contains translates of all compact subsets of $B_{Z}$. Using induction we construct an increasing sequence $\left\{X_{n}\right\}$ of closed separable subspaces of $Z$. Define $X_{1}:=Y$ and choose a dense countable subset $S_{1}$ of the unit ball of $X_{1}$. Choose a countable set $T_{1} \subset Z$ such that whenever $F$ is a finite subset of $S_{1}$ there exists $v \in T_{1}$ for which $v+F \subset C$. Choose a countable set $C_{1} \subset Z \backslash C$ such that $\overline{C_{1}} \supset C \cap X_{1}$. Suppose $X_{n}, S_{n}, T_{n}$ and $C_{n}$ for some $n \in N$ have been already constructed. Define

$$
X_{n+1}:=\overline{\operatorname{span}}\left(X_{n} \cup T_{n} \cup C_{n}\right),
$$

and choose a countable dense subset $S_{n+1} \supset S_{n}$ of the unit ball of $X_{n+1}$. Choose a countable set $T_{n+1} \subset Z$ such that whenever $F$ is a finite subset of $S_{n+1}$ there exists $v \in T_{n+1}$ for which $v+F \subset C$. Choose a countable set $C_{n+1} \subset Z \backslash C$ such that $\overline{C_{n+1}} \supset C \cap X_{n+1}$. Define

$$
X:=\overline{\bigcup_{n=1}^{\infty} X_{n}} \text { and } D:=\overline{\bigcup_{n=1}^{\infty}\left(X_{n} \cap C\right)} \text {. }
$$

The set $E:=\bigcup_{n=1}^{\infty} S_{n}$ is dense in $\bar{B}_{X}$ and from the construction it follows that any finite set contained in $E$ can be translated into $D$. The set $D$ is closed and convex, and it has empty interior because $\bigcup_{n=1}^{\infty} C_{n} \subset X \backslash D$ and $\overline{\bigcup_{n=1}^{\infty} C_{n}} \supset D$. An application of Theorem 3 completes the proof.

The following lemma is essentially due to James [4].

Lemma 5. Let $X$ be a nonreflexive Banach space. Then there exists a sequence $\left\{x_{n}\right\}$ in the unit ball of $X$ and $\varepsilon>0$ so that for any finite-dimensional subspace $Y$ of $X$ there exists $n \in N$ so that

$$
\operatorname{dist}\left(Y, \operatorname{co}\left\{x_{i}\right\}_{i=n}^{\infty}\right)>\varepsilon .
$$

Proof. The unit ball $\bar{B}_{X}$ of $X$ is not weakly compact, therefore by the GantmacherSmulyan theorem [3] there exists a decreasing sequence $\left\{C_{n}\right\}$ of nonempty, closed and convex subsets of $\bar{B}_{X}$ such that $\bigcap_{n=1}^{\infty} C_{n}=\emptyset$. We will show that there exist $\varepsilon>0$ and a decreasing sequence of convex nonempty sets $\left\{D_{n}\right\}$ so that $D_{n} \subset C_{n}$ for $n \in N$ and for any compact set $K \subset X$ there exists $m \in N$ such that

$$
(K+B(0, \varepsilon)) \cap D_{m}=\emptyset .
$$

Suppose for a contradiction that the required sequence $\left\{D_{n}\right\}$ does not exist. Let $C_{1, n}:=C_{n}$ for $n \in N$. There exists a compact convex set $K_{1}$ so that

$$
C_{1, n} \cap\left(K_{1}+B\left(0,2^{-1}\right)\right) \neq \emptyset \text { for } n \in N \text {. }
$$

Let $C_{2, n}:=C_{1, n} \cap\left(K_{1}+B\left(0,2^{-1}\right)\right)$ for $n \in N$. In general, if the sequence $\left\{C_{k, n}\right\}$ and the compact convex set $K_{k}$ have been already constructed, define

$$
C_{k+1, n}:=C_{k, n} \cap\left(K_{k}+B\left(0,2^{-k}\right)\right) \text { for } n \in N,
$$

and choose a compact convex set $K_{k+1}$ so that

$$
C_{k+1, n} \cap\left(K_{k+1}+B\left(0,2^{-(k+1)}\right)\right) \neq \emptyset \quad \text { for } n \in N .
$$

Then $C_{k+1, n} \subset C_{k, n}$, and by induction $C_{k, n+1} \subset C_{k, n}$. In particular if we define $G_{n}:=C_{n, n}$, then the sequence $\left\{G_{n}\right\}$ is decreasing, $G_{n} \subset C_{n}$ and

$$
G_{n+1} \subset K_{n}+B\left(0,2^{-n}\right) \text {. }
$$


Choose some $y_{n} \in G_{n}$. The sequence $\left\{y_{n}\right\}$ has a finite $\delta$-net for any $\delta>0$. Therefore it has a converging subsequence. The limit point of this subsequence is contained in $\bigcap_{n=1}^{\infty} C_{n}$, which is a contradiction. Now that we have shown the existence of the sequence $\left\{D_{n}\right\}$, to finish the proof simply choose any $x_{n} \in D_{n}$.

Theorem 6. Let $Z$ be a Banach space. The following are equivalent:

(i) $Z$ is not reflexive;

(ii) there exist a nontrivial closed subspace $X$ of $Z$ and a convex closed subset $Q$ of $X$ with empty interior which contains translates of all compact sets in $X$, i.e. whenever $K$ is a compact subset of $X$ there exists $x_{K} \in X$ so that $K+x_{K} \subset Q$.

Moreover, if $Z$ is separable, then (ii) holds with $X=Z$.

Proof. To show that (i) implies (ii) choose any separable nonreflexive subspace $X$ of $Z$; such a space exists by the Eberlein-Smulyan theorem. If $Z$ is separable let $X:=Z$. Choose an increasing sequence $\left\{X_{n}\right\}$ of finite-dimensional subspaces of $X$ so that $X=\overline{\bigcup_{n=1}^{\infty} X_{n}}$. Choose a sequence $\left\{x_{n}\right\}$ in the unit ball of $X$ and $\varepsilon>0$ as in Lemma 5 . By passing to a subsequence of $\left\{x_{n}\right\}$ if necessary we may suppose that

$$
\operatorname{dist}\left(\operatorname{span}\left(X_{n} \cup\left\{x_{i}\right\}_{i=1}^{n}\right), \operatorname{co}\left\{x_{i}\right\}_{i=n+1}^{\infty}\right)>\varepsilon \text { for } n \in N .
$$

Put $K_{n}:=X_{n} \cap B_{Z}$, and define

$$
D:=\overline{\mathrm{co}} \bigcup_{i=1}^{\infty}\left(x_{i}+(\varepsilon / 4) K_{i}\right) .
$$

The convex, closed and bounded set $\tilde{D}:=(4 / \varepsilon) D$ contains a translate of any finite subset of $\bar{B}_{X} \cap \bigcup_{n=1}^{\infty} X_{n}$. By Theorem 3, it only remains to show that the interior of $\tilde{D}$ is empty. For a contradiction, suppose that the interior of $D$ is nonempty. Because co $\bigcup_{i=1}^{\infty}\left(x_{i}+(\varepsilon / 4) K_{i}\right)$ is dense in $D$ there exist $n \in N, \alpha_{i} \geq 0$ and $u_{i} \in$ $(\varepsilon / 4) K_{i}, i=1, \ldots n$, so that $\sum_{i=1}^{n} \alpha_{i}=1$ and the point $z:=\sum_{i=1}^{n} \alpha_{i}\left(x_{i}+u_{i}\right)$ is contained in the interior of $D$. From (6) it follows that there exists a point $x^{*}$ in the unit sphere of $X^{*}$ so that

$$
\begin{array}{ll}
\left\langle x^{*}, x\right\rangle=0 & \text { for } \quad x \in X_{n}, \\
\left\langle x^{*}, x\right\rangle \leq-\varepsilon / 2 & \text { for } \quad x \in \operatorname{co}\left\{x_{i}\right\}_{i=n+1}^{\infty} .
\end{array}
$$

Choose a point $w$ in the unit sphere of $X$ for which

$$
\left\langle x^{*}, w\right\rangle \geq 1 / 2 \text {. }
$$

Since $z$ is an interior point of $D$, there exists an $r>0$ so that $z+r w \in D$. Consequently, there exist $m \in N, m>n, \beta_{i} \geq 0$ and $v_{i} \in(\varepsilon / 4) K_{i}, i=1, \ldots m$, so that $\sum_{i=1}^{m} \beta_{i}=1$ and if we define $y:=\sum_{i=1}^{m} \beta_{i}\left(x_{i}+v_{i}\right)$, then

$$
\|z+r w-y\|<r / 2 \text {. }
$$

From the definition of $x^{*}$ it follows that

$$
\begin{aligned}
& \left\langle r w+z-y, x^{*}\right\rangle \\
& =r\left\langle w, x^{*}\right\rangle+\left\langle\sum_{i=1}^{n} \alpha_{i}\left(x_{i}+u_{i}\right)-\beta_{i}\left(x_{i}+v_{i}\right), x^{*}\right\rangle-\left\langle\sum_{i=n+1}^{m} \beta_{i}\left(x_{i}+v_{i}\right), x^{*}\right\rangle \\
& \geq r / 2+0-\sum_{i=n+1}^{m} \beta_{i}\left(\left\langle x_{i}, x^{*}\right\rangle+\left\langle v_{i}, x^{*}\right\rangle\right) \\
& \geq r / 2-\sum_{i=n+1}^{m} \beta_{i}(-\varepsilon / 2+\varepsilon / 4) \\
& \geq r / 2
\end{aligned}
$$

which is a contradiction. 
Now, let us prove that (ii) implies (i). By Corollary 4, we may suppose that $X$ is separable. We will show that $X$ is nonreflexive and therefore $Z$ is also nonreflexive. For a contradiction suppose that $X$ is reflexive. Choose a sequence $\left\{x_{i}\right\}_{i=1}^{\infty} \subset X$ that is dense in the unit sphere of $X$. Denote

$$
K_{n}:=\operatorname{span}\left\{x_{i}\right\}_{i=1}^{n} \cap \bar{B}_{X}
$$

Clearly $\left\{K_{n}\right\}$ is an increasing sequence of compact subsets of the unit ball of $X$ for which

$$
\overline{\bigcup_{n=1}^{\infty} K_{n}}=\bar{B}_{X} .
$$

By Theorem 3 there exists a closed, convex and bounded subset $C$ of $X$ with empty interior which contains translates of all compact subsets of the unit ball of $X$. For $n \in N$ choose $z_{n} \in X$ so that $z_{n}+K_{n} \subset C$. The sequence $\left\{z_{n}\right\}$ is bounded, therefore it has a weakly converging subsequence $\left\{z_{n_{k}}\right\}$. Denote $z:=w$ - $\lim _{k \rightarrow \infty} z_{n_{k}}$. Because the set $C$ is convex and closed, it is also weakly closed. Consequently, because the sets $K_{n}$ are increasing, if there exists a $k \in N$ so that $y \in K_{n_{k}}$, then $y+z \in C$. Hence,

$$
z+\bar{B}_{X}=z+\overline{\bigcup_{k=1}^{\infty} K_{n_{k}}} \subset C,
$$

which, of course, means that the interior of $C$ is nonempty, which is a contradiction.

\section{ACKNOWLEDGMENT}

The authors thank the participants of the semiannual $\mathrm{k} \& \mathrm{k}$ analysis meeting and namely L. Zajíček for helpful discussions. We would also like to thank the referee for his close reading of the first submitted version of this paper.

\section{REFERENCES}

1. J.M. Borwein and D. Noll, Second order differentiability of convex functions in Banach spaces, Trans. Amer. Math. Soc. 342 (1994), 43-81. MR 94e:46076

2. J.P.R. Christensen, Topology and Borel structure, North-Holland, Amsterdam, 1974. MR 50:1221

3. M.M. Day, Normed linear spaces, Springer-Verlag, Berlin, 1973. MR 49:9588

4. R.C. James, Weak compactness and reflexivity, Israel J. Math. 2 (1964), 101-119. MR 31:585

Department of Mathematical Analysis, Charles University, Sokolovská 83, CZ-18600 Prague, Czech Republic

E-mail address: eva@csmat.karlin.mff.cuni.cz

Institut für Mathematik, Johannes Kepler Universität, Altenbergerstrasse, A-4040 Linz, Austria

E-mail address: stegall@caddo.bayou.uni-linz.ac.at 